\title{
Spectral width of SuperDARN echoes: measurement, use and physical interpretation
}

\author{
P. V. Ponomarenko ${ }^{1}$ and C. L. Waters ${ }^{1,2}$ \\ ${ }^{1}$ University of Newcastle, Callaghan, New South Wales, Australia \\ ${ }^{2} \mathrm{CRC}$ for Satellite Systems, Australia
}

Received: 20 July 2005 - Revised: 26 October 2005 - Accepted: 28 November 2005 - Published: 7 March 2006

\begin{abstract}
The Doppler velocity and spectral width are two important parameters derived from coherent scatter radar systems. The Super Dual Auroral Radar Network (SuperDARN) is capable of monitoring most of the high latitude region where different boundaries of the magnetosphere map to the ionosphere. In the past, the spectral width, calculated from SuperDARN data, has been used to identify the ionosphere footprints of various magnetosphere boundaries. In this paper we examine the way the spectral width is presently estimated from the radar data and describe several recommendations for improving the algorithm. Using the improved algorithm, we show that typical spectral width values reported in the literature are most probably overestimated. The physical interpretation of the cause of various magnitudes of the spectral width is explored in terms of the diffusion and dynamics of ionospheric plasma irregularities.
\end{abstract}

Keywords. Ionosphere (Ionospheric irregularities; Instruments and techniques) - Magnetospheric physics (Magnetosphere-ionosphere interactions)

\section{Introduction}

The Super Dual Auroral Radar Network (SuperDARN) is an international scientific consortium that operates and maintains high frequency (HF), over-the-horizon radars for conducting research in ionosphere and space physics (Greenwald et al., 1995). SuperDARN is designed to map ionospheric plasma convection over the auroral and polar cap regions. To achieve this, 2-D Doppler velocity $\left(V_{D}\right)$ vectors are estimated from pairs of radars with overlapping fields of view. In normal mode each beam is formed under computer control by a phasing matrix, to scan over 16 sequential beam directions. This provides an overall azimuth coverage of $\simeq 50^{\circ}$. A multi-pulse transmit sequence enables SuperDARN radars to measure Doppler velocities up to $2 \mathrm{~km} / \mathrm{s}$ over distances of $\leq 3500 \mathrm{~km}$ (Greenwald et al., 1985; Hanuise

Correspondence to: P. V. Ponomarenko

(phpp@alinga.newcastle.edu.au) et al., 1993; Baker et al., 1995; Barthes et al., 1998). The resulting autocorrelation function (ACF) for every range gate is used to estimate $V_{D}$, the scattered signal power and the spectral width, $W$. The Doppler velocity is derived from the temporal gradient of the ACF phase. The spectral width is estimated from the ACF power decorrelation time. These parameters are calculated at each radar site by a computer algorithm known as FITACF, which, among other tasks, fits model functions to various experimental ACF characteristics.

Since the late 1990s, the spectral width has been widely used to infer ionospheric footprints of various magnetospheric boundaries, such as the cusp (Baker et al., 1995) and open-closed magnetic field line boundary (OCB) (Dudeney et al., 1998). Cusp echoes are usually characterised by a relatively large spectral width, $W \geq 200 \mathrm{~m} / \mathrm{s}$, while equatorward of the cusp it appears to be limited to $W \leq 50 \mathrm{~m} / \mathrm{s}$ (Baker et al., 1995). Night-time radar returns regularly exhibit a boundary around $70^{\circ}$ MLAT (e.g. Parkinson et al., 2004), which is accompanied by a similar change in $W$. A recent statistical study by Chisham et al. (2004) confirmed previous case study results, that the spectral width is a good proxy for the OCB over most of the nighttime sector.

Using the spectral width to identify magnetosphere boundaries mapped onto the ionosphere appears to have become standard procedure, as shown, for example, by the extensive referencing on this topic by Chisham et al. (2004). Despite this, large spectral width values ( $W \geq 200 \mathrm{~m} / \mathrm{s}$ ) obtained from SuperDARN radars still lack an adequate physical interpretation. Ponomarenko and Waters (2003) have briefly discussed some of the physical factors that affect the spectral width. In general, these factors are related to either the ionospheric drift velocities or the lifetime of the irregularities. The fastest process determines the ACF correlation time, $\tau_{c}$, and therefore the spectral width, $W \sim 1 / \tau_{c}$. There have been several attempts to clarify factors that control the measured spectral width. The most general approach, based on a combination of the random velocity distribution of electron density irregularities and ionospheric diffusion was used by Hanuise et al. (1993) and Villain et al. (1996). They found that for large $W$ the value of $\tau_{c}$ depends on the average irregularity 


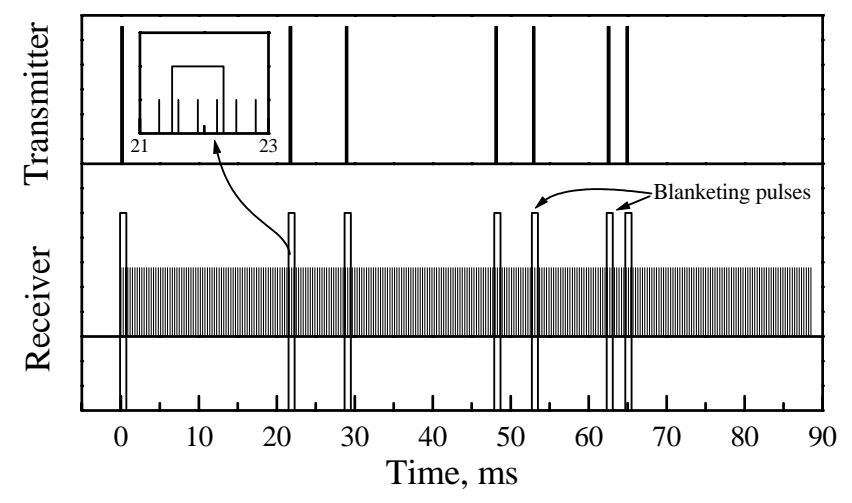

Fig. 1. Transmit pulse sequence and receiver sampling times used in SuperDARN radars.

lifetime, and the ACF power, as a function of lag, is exponential in shape, regardless of the type of the irregularity decay process. However, estimates of the plasma diffusion coefficient, $D \simeq 100-200 \mathrm{~m}^{2} / \mathrm{s}$, derived from the measured spectral width values, were much larger than typical values for the ambipolar diffusion coefficient in the F-region, which are $D \sim 1 \mathrm{~m}^{2} / \mathrm{s}$ (e.g. Hysell et al., 1996). André et al. (2000) discussed Bohm diffusion as a possible alternative to the classical mechanism. The semiempirical expression for the Bohm diffusion coefficient is (Chen, 1984)

$D_{B}=k T / 16 e B$,

where $k$ is Boltzmann's constant, $T$ temperature, $e$ electron mass and $B$ magnetic field. Equation (1) predicts values of $D_{B} \sim 200 \mathrm{~m}^{2} / \mathrm{s}$ for typical ionospheric conditions, while many of the experimental estimates exceed $300 \mathrm{~m}^{2} / \mathrm{s}$.

Another interpretation was based on small-scale electric field fluctuations that produce random velocity variations in the cusp region (Baker et al., 1995). However, in order to be a viable mechanism, the electric fields need to be rather large at around a few $\mathrm{mV} / \mathrm{m}$.

Explanations for large values of the spectral width have also considered possible violations of the assumptions used to develop the FITACF algorithms. For example, multicomponent ACFs have been reported in the large- $W$ sections of backscatter data from the cusp (Baker et al., 1995; Moen et al., 2000). These authors suggested that since the algorithm for estimating the spectral width assumes a single component ACF, multi-component ones may cause an overestimation and high variability of $W$. In a study by Moen et al. (2000) the frequency spectra were obtained via the Fourier transform of the experimental ACF values, assuming that the cross-range interference was negligible. However, the effect of this interference was recognised by Baker et al. (1995), who attempted to remove contaminated ACF lags but still observed multi-peak spectra in cusp echoes.

André et al. (1999) suggested that large spectral width values might be caused by a nonlinear response of the SuperDARN autocorrelation routine to Pc1-2 ULF waves with periods smaller than the integration time $t_{i} \simeq 1-7 \mathrm{~s}$. To support this, the authors modelled a ULF-modulated radar time series and processed these with the standard FITACF package. They obtained large spectral width values even for relatively small-amplitude waves. However, the reported nonlinear response in the correlation process prompted Ponomarenko and Waters (2003) to examine these results. The modelled spectral broadening in André et al. (1999) was found to be caused by a mathematical error.

Among other possible causes for large spectral width values, André et al. (2000) considered large- and meso-scale velocity gradients within the scatter volume. However, their model calculations showed that these factors were insufficient for producing either large $W$ values or multi-component ACFs.

The generation of large spectral width values via restructuring of the ionospheric plasma at decameter scale lengths by particle precipitation was proposed by Moen et al. (2000). Furthermore, Chisham et al. (2005) established inverse proportionality between $W$ and precipitating electron energy flux measured by DMSP satellites. To explain this fact, the authors assumed that the large spectral width values are produced by the small-scale convection electric field structures in the vicinity of the OCB, but they become effectively suppressed equatorward of the OCB by the increased ionospheric conductivity produced by the high electron energy fluxes (Parkinson et al., 2004).

The effect of forward scatter of the HF waves from the radar by ionospheric turbulence on $W$ was modelled by Valliéres et al. (2004), but this mechanism seems to be only capable of producing large values of $W$ at operating frequencies close to the critical frequency of the ionospheric F-region.

Despite the number of proposed mechanisms, it is unclear if any can explain routinely observed spectral width values of $W \geq 200 \mathrm{~m} / \mathrm{s}$ reported in the literature. With this in mind, we examined the estimation of spectral width in the data processing procedures used by all SuperDARN radars as coded in the latest available version (1.09) of the FITACF software. In Sect. 2 we outline the FITACF routines that affect spectral width estimates and describe several modifications that improve the calculation. Analytical functions for fitting the ACF are examined in Sect. 3 within the context of the physics involved in the HF scatter. A representative data set from the TIGER (Tasmania) radar is used to illustrate the effects of the modifications to FITACF. In Sect. 4 we examine physical explanations for the variation in magnitude of the spectral width computed from SuperDARN data.

\section{Multi-pulse radars and the ACF}

SuperDARN is required to measure $V_{D}$ up to $\sim 1-2 \mathrm{~km} / \mathrm{s}$ over distances up to $d_{\text {max }} \simeq 3500 \mathrm{~km}$. This would be impossible using a single-pulse transmit scheme, where the pulse repetition frequency derived from the maximum return range would be $f_{r} \leq 40 \mathrm{~Hz}$. Therefore, the maximum Doppler shift that can be measured with a single-pulse 


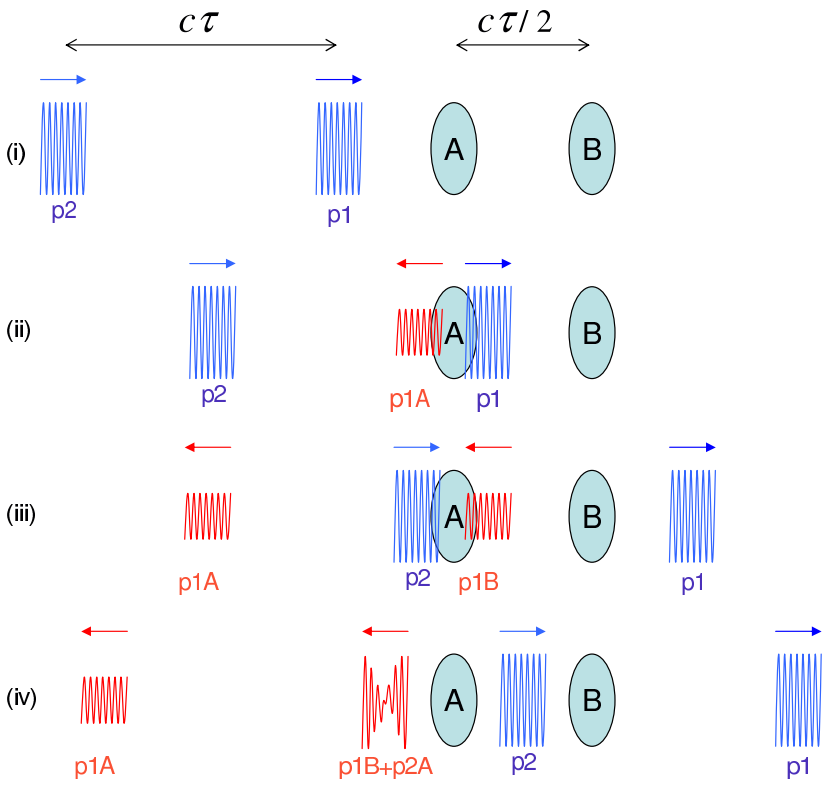

Fig. 2. Illustration of conditions for cross-range interference. Transmitter pulses are drawn in blue, reflected ones in red. Time increases from top to bottom.

scheme is equal to the Nyquist frequency $f_{n}=f_{r} / 2 \leq 20 \mathrm{~Hz}$, while ionospheric convection can produce Doppler shifts up to $f_{D}^{\max }=2 V_{D}^{\max } / \lambda \sim 100-200 \mathrm{~Hz}$.

In order for SuperDARN to measure the required $V_{D}$ values, a seven-pulse coded sequence was implemented (Greenwald et al., 1985; Hanuise et al., 1993; Baker et al., 1995; Barthes et al., 1998). It produces ACFs with an elementary lag of $2.4 \mathrm{~ms}\left(f_{n} \simeq 200 \mathrm{~Hz}\right)$. The transmit pulse length of $300 \mu$ s gives a spatial resolution of $45 \mathrm{~km}$ over $70-75$ range gates (maximum range $3550 \mathrm{~km}$ ).

The multi-pulse transmit sequence overlaid with the receiver samples is shown in Fig. 1. The receiver data is sampled at $300 \mu$ s intervals for a time that allows the last pulse from the sequence to arrive from the furthest range ( $\Delta t_{\max } \simeq 0.1 \mathrm{~s}$ ). The ACF is computed from the 17 possible time lags available via the combinations of pairs of pulses, giving a maximum lag value of $\tau_{\max }=43.2 \mathrm{~ms}$. For each beam, the process is repeated for the specified integration time, $t_{i} \simeq 3-7 \mathrm{~s}$. This sets the number of ACFs that are averaged, $N_{\text {avg }}=t_{i} / \Delta t_{\max }$ (usually $N_{\text {avg }} \simeq 70$ ). The timing of data sampled at the receiver sometimes coincides with the time a transmit pulse must be sent. For this case, the receiver is "blanketed" around that time for a duration of 2-3 pulse lengths (see insert in Fig. 1). This effect is easily identified from the radar timing information and the appropriate samples are rejected. While the multi-pulse transmit scheme allows measurement of larger $V_{D}$, it has some disadvantages. The major problem is cross-range interference (CRI), when returns from different pulses in the sequence arrive at the receiver simultaneously from different ranges. Figure 2 illustrates CRI for four consecutive time snapshots (i)-(iv) of two pulses, $p 1$ and $p 2$, transmitted time $\tau$ apart and propagating

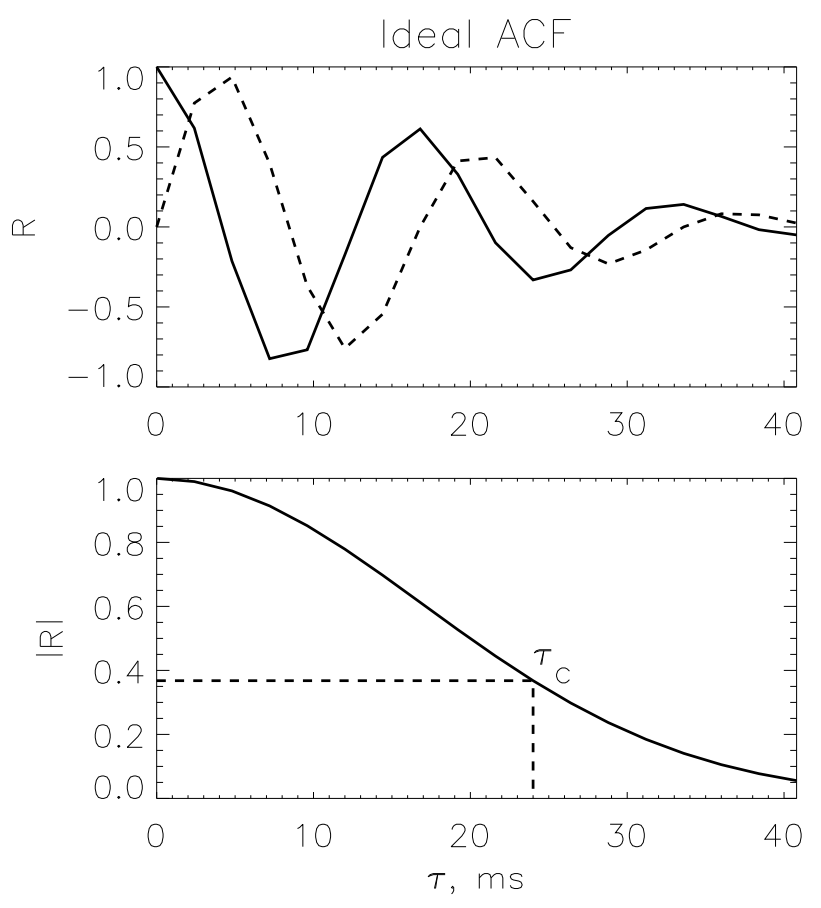

Fig. 3. Ideal ACF characteristics from SuperDARN radars. (Top) The real part (solid) and imaginary part (dashed) of a normalised, ideal ACF. The ACF magnitude (bottom) showing the correlation time scale, $\tau_{c}$, which is used to calculate spectral width, $W$.

from left to right. The pulses approach two individual targets $A$ and $B$ separated by $c \tau / 2$, where $c$ is the speed of light (i). After the first pulse, $p 1$, reaches the first target, $A$, it produces an echo $p 1 A$, which propagates back to the radar (ii). In the same way $p 1$ also generates a second echo, $p 1 B$, which reaches target $A$ from the right at the same time as the second transmitter pulse $p 2$ arrives there from the left and generates another echo, $p 2 A$ (iii). From that point, both echoes, $p 1 B$ and $p 2 A$, travel back to the receiver, interfering with each other (iv).

In ionospheric sounding, a plasma volume with intensive small-scale irregularities presents a continuous target that may stretch over $\leq 1000 \mathrm{~km}$, providing favourable conditions for CRI. An excellent illustration of CRI is presented in Fig. 1F of Yukimatu and Tsutsumi (2002), which shows how the echoes received from all seven pulses overlap in the time domain. For a known multi-pulse sequence, it is quite straightforward to calculate when and at which ACF lags CRI might occur. However, it is a more complicated matter to detect if CRI is significant for a given range. This aspect of the ACF processing is discussed further in Sect. 3.

To estimate $W$ and $V_{D}$, the data processing algorithms in FITACF deal with power and phase calculated from real and imaginary parts of a complex ACF, $R(\tau)$. Figure 3 shows a modelled single-frequency ACF (top). The real part of the ACF, $\Re[R(\tau)]$, is an even function with a maximum at zero lag, and the imaginary part, $\Im[R(\tau)]$, is an odd function of the lag. Both the real and imaginary parts of the ACF 

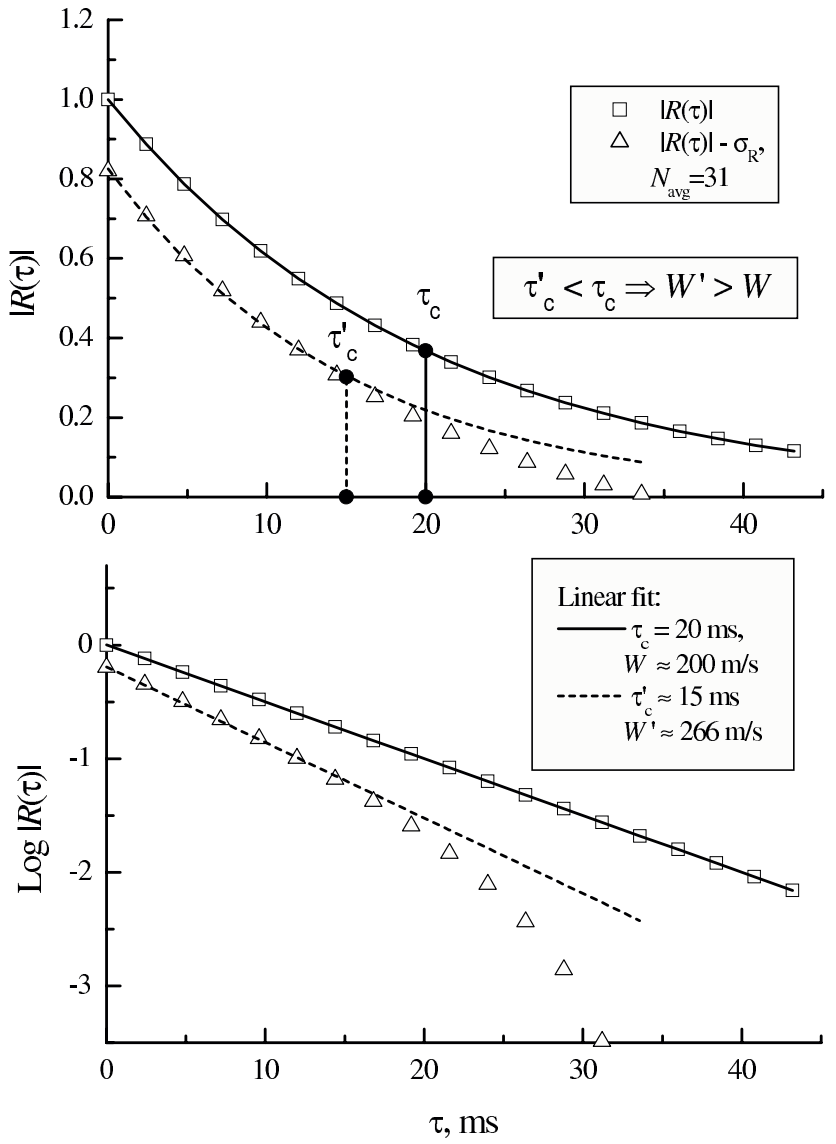

Fig. 4. Illustration of the overestimation of $W$ due to the extraction of the statistical fluctuation level, $\sigma_{r}=R(0) / \sqrt{N_{a v g}}$, from ACF power in FITACF.

decay in amplitude with increasing lag as the received data decorrelate. This is illustrated in the bottom panel of Fig. 3, which shows the ACF power $|R(\tau)|=\sqrt{\mathfrak{R}^{2}[R(\tau)]+\Im^{2}[R(\tau)]}$ used to estimate lag zero power and spectral width $W \sim 1 / \tau_{c}$ via fitting an exponential or Gaussian model. The fitting procedure is based on the assumption that the Fourier transform of the ACF has a single spectral component, i.e. $|R(\tau)|$ decreases with increasing lag. The ACF phase, $\phi(\tau)=\operatorname{atan}\left\{\Im^{2}[R(\tau)] / \Re^{2}[R(\tau)]\right\}$ is used to calculate $V_{D}$. Further details and illustrations on the fitting procedures may be found in Hanuise et al. (1993), Baker et al. (1995), and Barthes et al. (1998).

\section{Calculation of spectral width}

The FITACF software used routinely by all SuperDARN radars consists of a number of subroutines and those relevant to this study are described in Appendix A. The major tasks performed by FITACF are to:

1. Determine ACF lags contaminated by cross-range and pulse-overlap interference and exclude them from further processing.
2. Find lag zero noise, $R_{n}(0)$, and non-zero lag noise, $\left\langle R_{n}(\tau \neq 0)\right\rangle$, using the ACFs with the lowest lag zero power.

3. Use the above noise levels to check for any coherent interference, i.e. "noise ACF". If $\left\langle R_{n}(\tau \neq 0)\right\rangle$ exceeds the expected fluctuation level, $R(0) / \sqrt{N_{\text {avg }}}$, by $\geq 60 \%$, then the "noise ACF" is estimated and subtracted from all ACFs.

4. If the "noise ACF" was subtracted, then $R_{n}(0)$ and $\left\langle R_{n}(\tau \neq 0)\right\rangle$ are recalculated for the "cleaned" ACFs.

5. Subtract fluctuation level, $\sigma_{R}=R(0) / \sqrt{N_{\text {avg }}}$, and $\left\langle R_{n}(\tau \neq 0)\right\rangle$ from the ACF power. All ACFs with a negative zero lag power are excluded. All non-zero ACF lags with negative power or those considerably deviating from a single-component ACF shape are marked as "bad lags" and excluded from power fitting.

6. $W$ is estimated by fitting linear or quadratic functions to the logarithm of the ACF power, for exponential or Gaussian models, respectively, with contributions from different lags weighted by the linear ACF power.

We found that a number of processes currently implemented in the code adversely affect the estimation of the spectral width. These processes and recommendations for changing the algorithms are summarised below.

\subsection{Fluctuation level}

Firstly, we focus on the use of the statistical fluctuation level, $\sigma_{R}=R(0) / \sqrt{N_{\text {avg }}}$. In the original FITACF package, this value is treated as a positive offset to all ACF lags and is subtracted from the ACF power, $|R(\tau)|$, before applying the fitting routines. All resulting ACF lags with negative power are effectively excluded from the power fitting process. We would like to point out that by definition $\sigma_{R}$ is a magnitude of statistical fluctuations of the measured ACF power around its expectation value. These fluctuations can both increase and decrease the power, so they cannot be treated as a positive offset. With subtraction of $\sigma_{R}$ the ACF power becomes biased towards lower values, and this bias increases with decreasing $N_{\text {avg }}$. This, in turn, leads to a systematic bias of $W$ towards larger values. We illustrate this effect in Fig. 4, where we reproduce the FITACF estimation of $W$ for a model ACF with an exponential $|R(\tau)|$, characterized by a correlation time $\tau_{c}=20 \mathrm{~ms}$ (squares). We used the same $N_{\text {avg }}=31$ and transmitter frequency, $f_{0}=11.9 \mathrm{MHz}$ as for the specialmode experimental data presented later. The above values for $\tau_{c}$ and $f_{0}$ correspond to $W \simeq 200 \mathrm{~m} / \mathrm{s}$. In Fig. 4 triangles show ACF power with the subtracted fluctuation level, $|R(\tau)|-\sigma_{R}$ (lags with negative ACF power are ignored). In FITACF, $W$ is estimated by fitting linear or quadratic functions to the logarithm of the ACF power for exponential and Gaussian models, respectively. During the fitting procedure the results are weighted by the ACF power. In the bottom panel of Fig. 4 we show results of a linear fit applied 

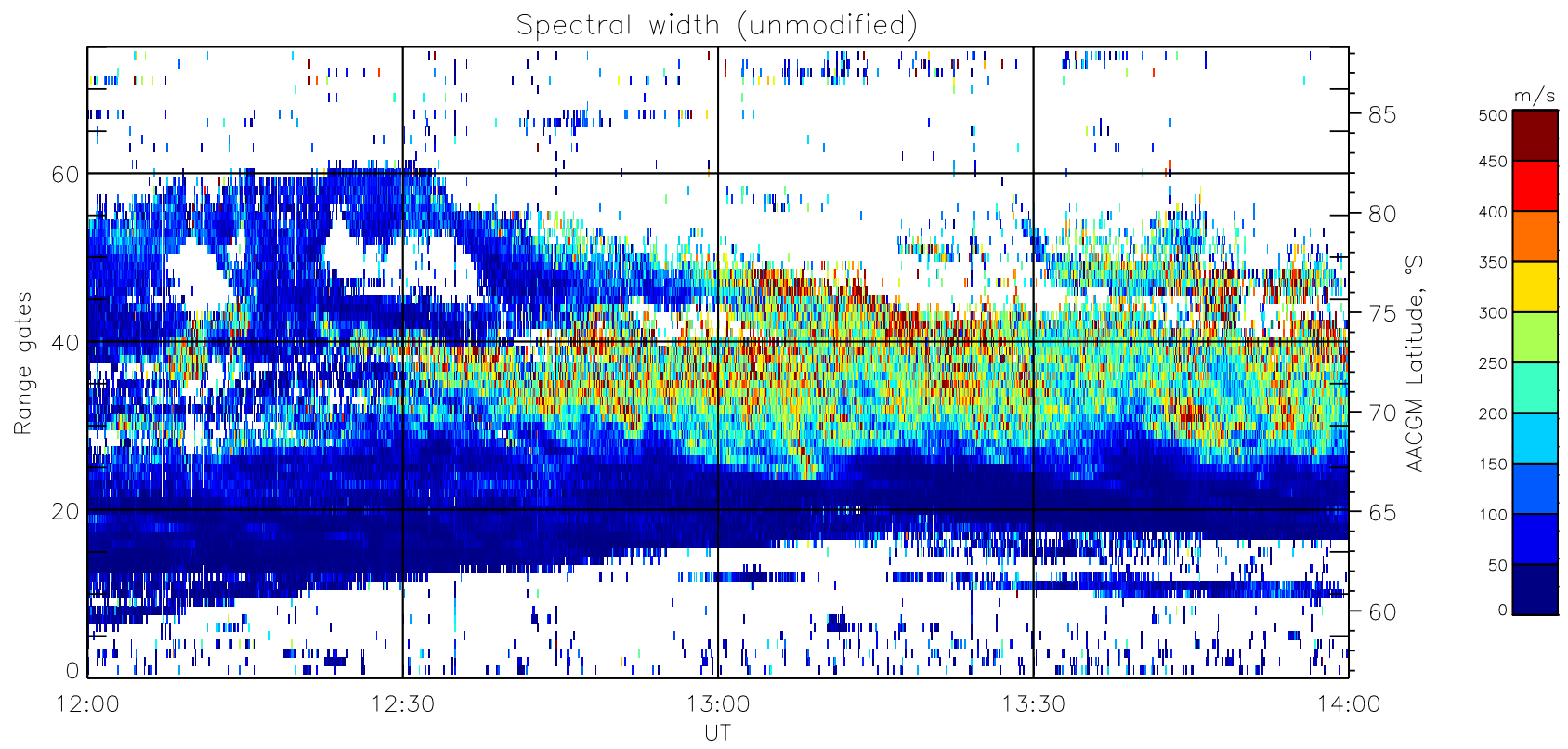

Fig. 5. Spectral width for 12:00-14:00 UT on 10 December 1999, obtained from TIGER beam \#4 using the original FITACF procedure.
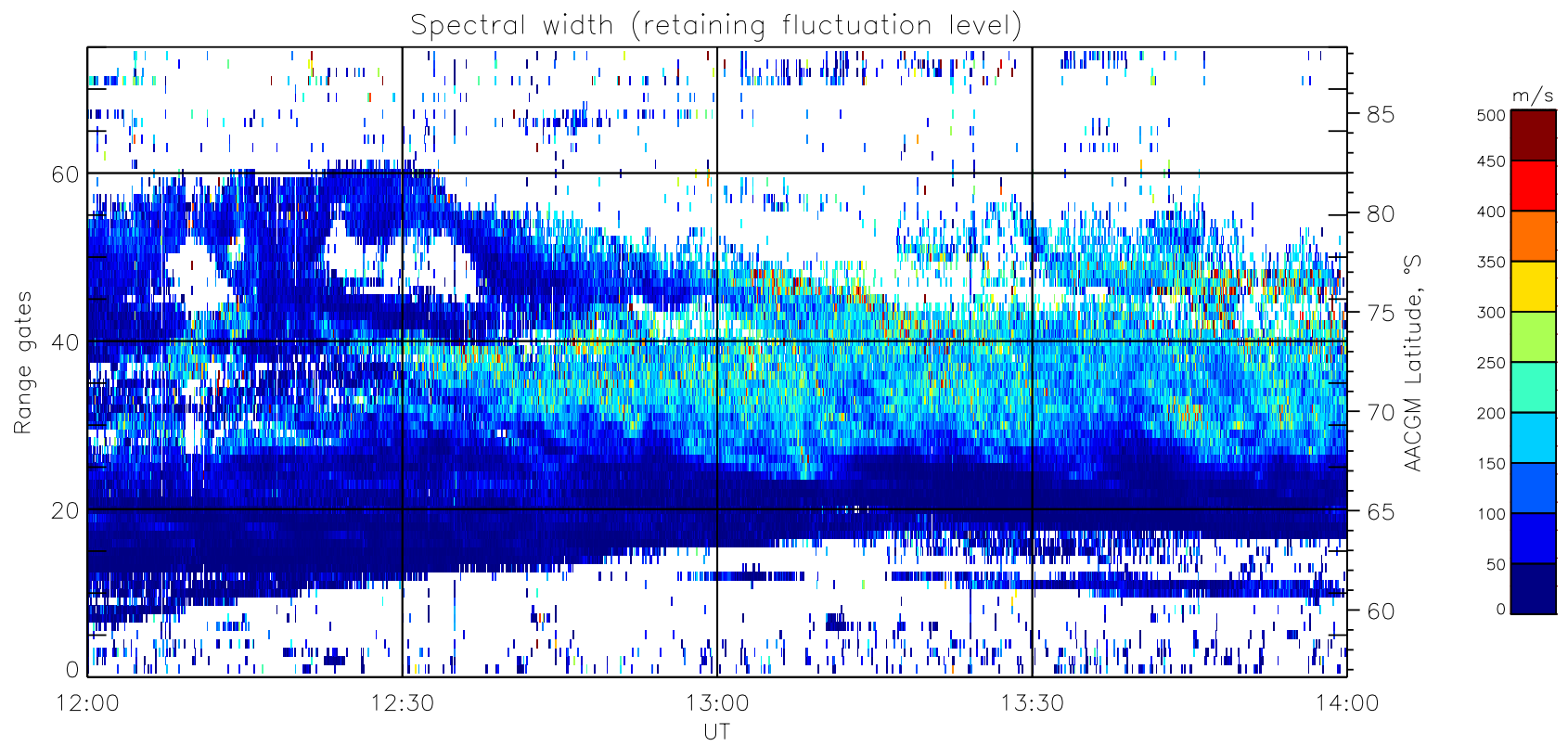

Fig. 6. The same data as Fig. 5 but without extracting the fluctuation level $\sigma_{R}$ from the ACF power $|R(\tau)|$ before the curve fitting process.

to both $\log |R(\tau)|$ (solid line) and $\log \left(|R(\tau)|-\sigma_{R}\right.$ ) (dashed line). The same curves are also shown on a linear scale in the top panel, together with the respective $e$-folding time scales, $\tau_{e}$ and $\tau_{c}^{\prime}$. In this case the measured spectral width for the "modified" ACF power is $W^{\prime} \simeq 266 \mathrm{~m} / \mathrm{s}$, i.e. it exceeds the original value, $W \simeq 200 \mathrm{~m} / \mathrm{s}$, by $\simeq 33 \%$. Further modelling showed that for $N_{a v g}=31$ this increase remains close to $30 \%$ for $W=50-300 \mathrm{~m} / \mathrm{s}$. In normal operational mode SuperDARN radars use $N_{a v g} \simeq 70$, and in this case the average modelled increase in spectral width was close to $20 \%$ over the same width range.
To illustrate the effect of extracting $\sigma_{R}$ on real radar records, we analysed data obtained between 12:00 and 14:00 UT ( $\sim 23: 00-01: 00$ MLT) on 10 December 1999 from the TIGER (Tasman International GeoEnvironmental Radar) SuperDARN radar (Parkinson et al., 2004). Figure 5 shows a time-range map for $W$ obtained for this interval from data processed using the original, unmodified FITACF code. The radar was programmed for high temporal resolution with $\simeq 3$ s/sample $\left(N_{\text {avg }}=31\right)$ on beam \#4, which points towards the south AACGM Pole. Starting from 12:30 UT, the data show a pronounced boundary between low and 
TIGER Beam \#4, 12:00-14:00 UT, 10/12/1999

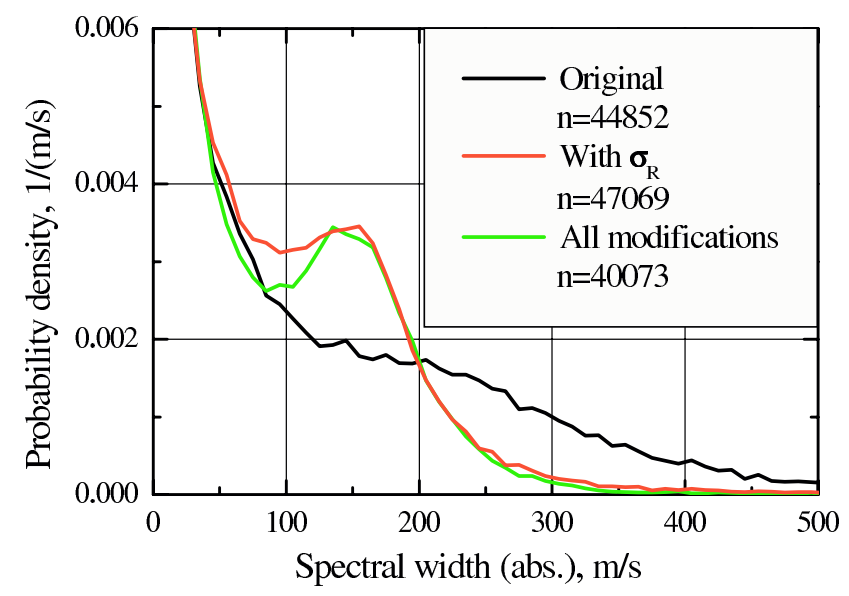

Fig. 7. Spectral width histograms for data presented in Fig. 5 (unmodified FITACF, black), Fig. 6 (without subtracting fluctuation level, red), and after applying all changes proposed in Sect. 3.4. Both modified algorithms essentially confine $W$ to $\sim 250 \mathrm{~m} / \mathrm{s}$.

TIGER Beam \#4, 12:00-14:00 UT, 10/12/1999

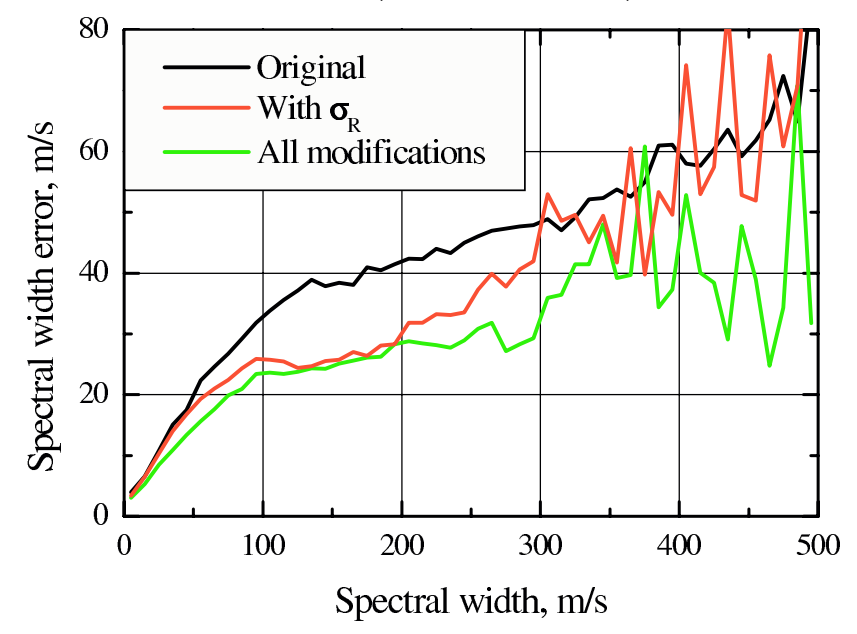

Fig. 8. The same as in Fig. 7 but for a spectral width fitting error.

high $W$ values around $\simeq 67-68^{\circ}$ MLAT. Sea/ground scatter echoes have not been removed from the data. All ACFs with less than three "good" lags were rejected.

In Fig. 6 the same data were re-processed with FITACF modified to not remove $\sigma_{R}$ before the curve fitting routines.

An overall decrease in the magnitudes of $W$ is clearly seen while the location of the sharp latitudinal gradient in $W$ remains essentially the same. This effect is quantified in Fig. 7, which shows histograms calculated from the original and modified data. The modified FITACF produces values for $W$ that are essentially confined to below $200 \mathrm{~m} / \mathrm{s}$ while the unmodified routine gives many values well beyond this limit. Furthermore, the modified FITACF generates considerably smaller fitting errors over the range $W=50-300 \mathrm{~m} / \mathrm{s}$, as shown in Fig. 8.

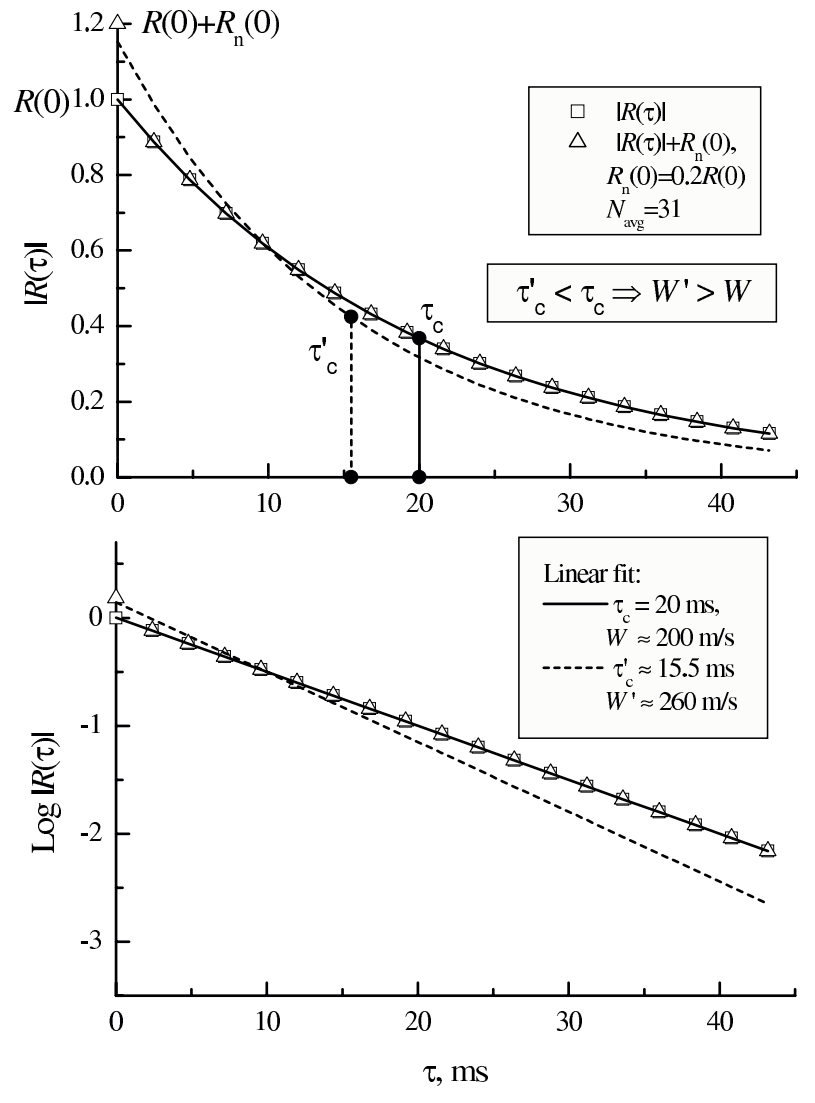

Fig. 9. The effect of zero-lag noise on estimating spectral width.

Finally, following the same arguments as for $\sigma_{R}$, we want to point out that there is no justification for FITACF extracting $\left\langle R_{n}(\tau \neq 0)\right\rangle$. Due to the fact that all coherent noise has already been removed (see point 3 at the beginning of this section and Sect. A2 in the Appendix), this parameter represents a fluctuation level for the incoherent background noise. However, its subtraction has little effect on the spectral width estimate compared with that caused by the removal of $\sigma_{R}$, so we left that part of the FITACF code unchanged.

\subsection{Zero lag noise}

The next modification to FITACF involved the zero lag noise, $R_{n}(0)$ (for details see Sect. A2 in the Appendix). Because all coherent noise has been removed from the ACFs, $R_{n}(0)$ represents a $\delta$-correlated addition to the ACF power at $\tau=0$. The presence of a $\delta$ function at $\tau=0$ effectively underestimates the correlation time, which causes an overestimation of the spectral width. This effect is illustrated in Fig. 9, where we used the same basic model as in Fig. 4 but modified it by adding $R_{n}(0)=0.2 R(0)$ at $\tau=0$. While the initial spectral width value is $W \simeq 200 \mathrm{~m} / \mathrm{s}$, the modified one is larger by $\simeq 30 \%, W^{\prime} \simeq 260 \mathrm{~m} / \mathrm{s}$.

Furthermore, our model calculations show that the combined effect of subtracting $\sigma_{R}\left(N_{\text {avg }}=31\right)$ and retaining $20 \%$ zero lag noise leads to a $100 \%$ overestimation of the spectral width, giving $W^{\prime} \simeq 400 \mathrm{~m} / \mathrm{s}$ for $W \simeq 200 \mathrm{~m} / \mathrm{s}$. 
The lag zero noise effect was mostly found to influence the sea/ground scatter rather than ionosphere scatter. To quantify the change in the experimental sea-scatter spectral width, we analysed data from TIGER beam \#4 recorded on 20:0022:00 UT on 28 September 2000, which consisted of seascatter echoes only (Ponomarenko et al., 2005). Comparing the original estimates of $W$ with the FITACF modified to extract the zero lag noise, we found that the original algorithm incorrectly classified up to $20 \%$ of the echoes as ionospheric rather than sea scatter.

\section{3 "Bad lag" routines}

The "bad lag" routines in FITACF (Sect. A3 in the Appendix) deal with the distortion of the ACF shape caused by both known and unknown factors. The "known" category contains a low signal-to-noise ratio, transmitter pulse overlap with receiver samples (Fig. 1), and CRI (Fig. 2). Bad lags caused by "unknown" factors are identified as a deviation of $|R(\tau)|$ from the single-component ACF shape required for fitting.

An analysis of "known" factors revealed that many ACF lags with high levels of CRI were not rejected by the original FITACF routines. To illustrate this, consider the situation shown in Fig. 2. Assume that the scattered signals from targets, $\mathrm{A}$ and $\mathrm{B}$, are given by

$u_{A}(t)=u_{A 0} e^{i\left\{\omega t+\psi_{A}(t)\right\}}$

and

$u_{B}(t)=u_{B 0} e^{i\left\{\omega t+\psi_{B}(t)\right\}}$

and are not correlated with each other. In this case the ACF calculated for the range $\mathrm{A}$ at lag $\tau$ consists of two terms:

$R_{A A}^{*}(\tau)=\left\langle\left[u_{A}(t)\right]\left[u_{A}(t+\tau)+u_{B}(t+\tau)\right]\right\rangle=$

$R_{A A}(\tau)+R_{A B}(\tau)$

where $\langle\ldots\rangle$ represents statistical averaging. Due to the fact that $u_{A}(t)$ and $u_{B}(t)$ are not correlated, the last (interference) term will reach zero for $N_{\text {avg }} \rightarrow \infty$. However, for a finite $N_{\text {avg }}$ this term produces an additional statistical fluctuation level (see Sect. A1 in the Appendix $), \quad \sigma_{R}^{*} \simeq u_{A 0} u_{B 0} / \sqrt{N_{\text {avg }}}=\sqrt{R_{A A}(0) R_{B B}(0) / N_{\text {avg }}}$, while the fluctuation level from $R_{A A}(\tau)$ is $\sigma_{R} \simeq u_{A 0}^{2} / \sqrt{N_{\text {avg }}}=R_{A A}(0) / \sqrt{N_{\text {avg }}}$. Therefore, any CRI left in the ACFs cause an increase in the overall statistical fluctuation level. Importantly, this interference can contribute to the ACF power, both constructively and destructively, producing either positive or negative "spikes". The expected fluctuation levels are equal $\left(\sigma_{R}^{*}=\sigma_{R}\right)$ when $R_{A A}(0)=R_{B B}(0)$. It is easy to show that CRI affects the same lag in $R_{B B}^{*}$ in the same way, which makes the last condition a natural threshold for rejecting CRI contaminated ACF lags: if $\mu=R_{B B}(0) / R_{A A}(0)>1$, then the lag $R_{A A}^{*}(\tau)$ is rejected; otherwise, we have to reject the same lag in $R_{B B}^{*}$.

In contrast, the CRI algorithm implemented in FITACF (Sect. A3) applies a much larger threshold, $\mu=0.3 N_{\text {avg }} \simeq 20$.

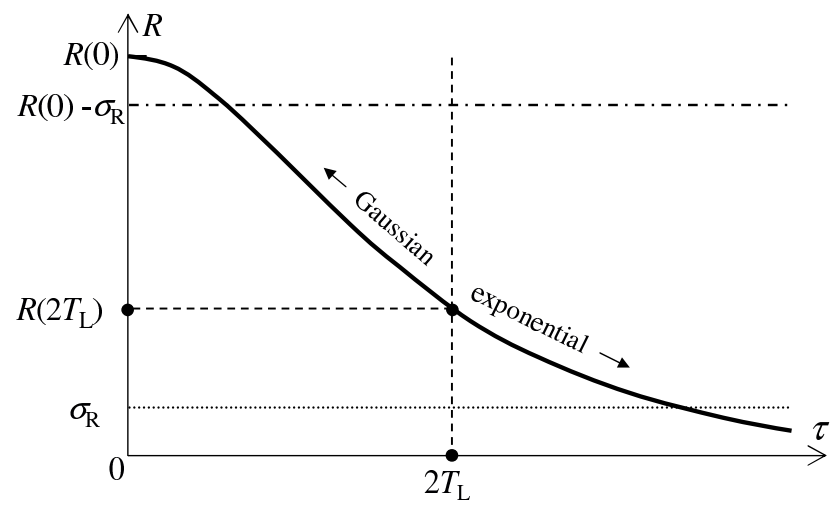

Fig. 10. Physical meaning of the Lagrangian correlation time, $T_{L}$, for the model described by Eq. (2).

This means that power from the "contaminating" range is allowed to be $\simeq 20$ times larger than power from the examined range. In normal operation mode $N_{\text {avg }} \simeq 70$, in which case the expected fluctuation level can be as large as $\sigma_{R}+\sigma_{R}^{*}=\sigma_{R}\left(1+\sqrt{0.3 N_{\text {avg }}}\right) \simeq 5.5 \sigma_{R}$. Consequently, many lags with unacceptable levels of CRI remain in the ACFs.

The ACF "shape" routines in FITACF are based on empirical criteria (Sect. A3 in the appendix) and are designed to account for significant ACF power variations from an unknown origin. Keeping this in mind, one might suspect that a considerable part of these deviations originates from an inadequate treatment of CRI, which is capable of causing both a negative and positive depletion of non-zero lag power. In fact, when we changed the threshold value for CRI rejection using the data set in Fig. 5 from $0.3 N_{\text {avg }}$ to 1 , the number of "bad shape" lags decreased by $\sim 65 \%$. This means that we could effectively bypass the "bad shape" FITACF routines (Sect. A3). Importantly, after using the lower CRI threshold $(\mu=1)$, the effective fluctuation level in the ACF lags can be as high as $\simeq 2 \sigma_{R}$ compared with $\simeq 5.5 \sigma_{R}$ from the original FITACF.

\subsection{Summary of FITACF Modifications}

We revised a number of FITACF routines that deal with noise and interference, and that affect spectral width estimates, both directly and indirectly. We established that the largest effect is caused by the removal of the statistical fluctuation level, $\sigma_{R}$, from the ACF power. Figures 5-7 show that this causes considerable overestimation of $W$, spawning searches for physical mechanisms that may cause these artificially large spectral widths. We have implemented the following modifications to FITACF:

1. To not remove the statistical fluctuation level from the ACF power.

2. To extract the zero lag noise from the ACF power.

3. To decrease the cross range interference threshold from $\mu=0.3 N_{\text {avg }}$ to $\mu=1$. 


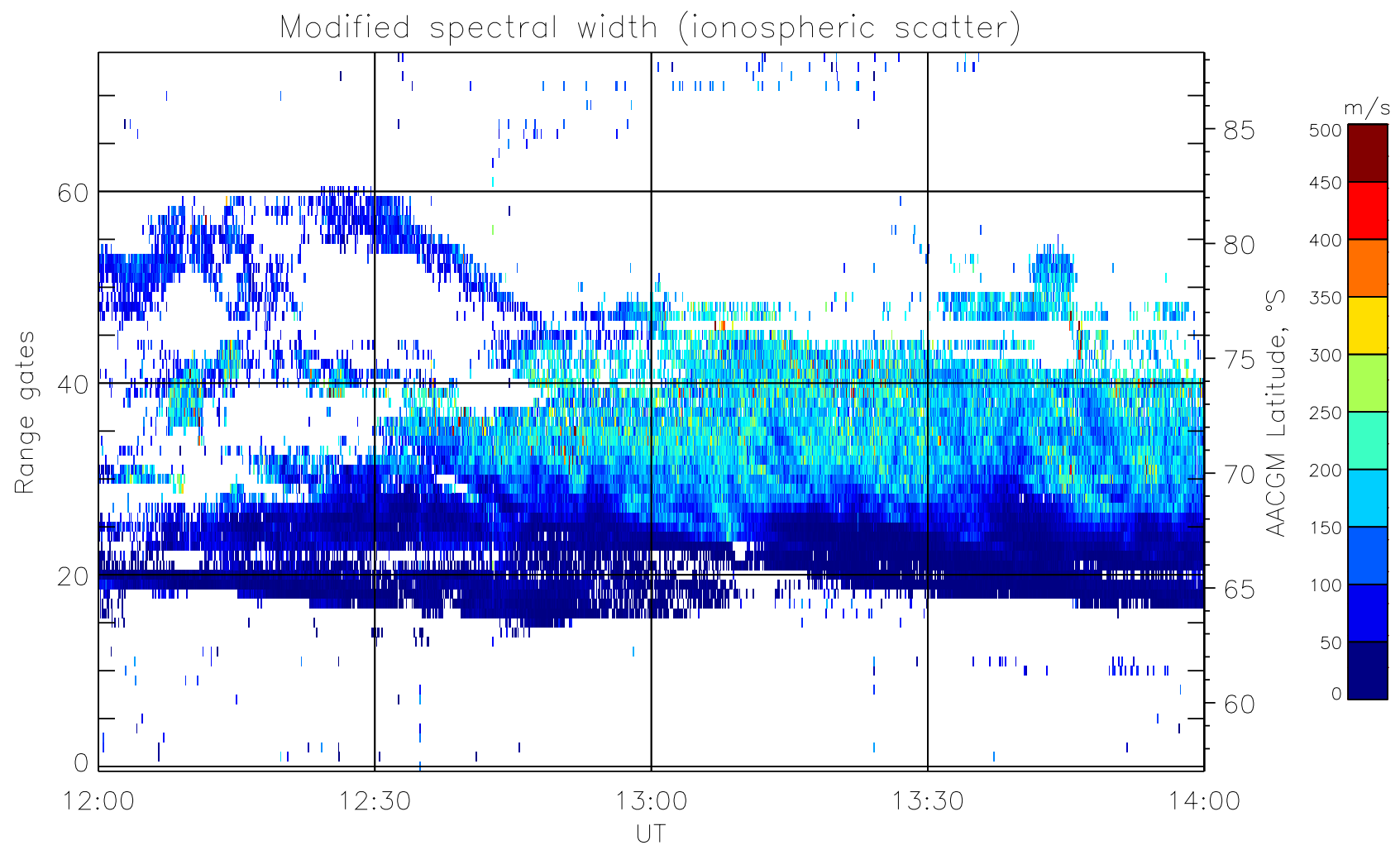

Fig. 11. Spectral width of the ionospheric scatter data from TIGER beam \#4 over 12:00-14:00 UT on 10 December 1999. The data are from the modified FITACF routines (see Sect. 2 for details).

TIGER Beam \#4, 12:00-14:00 UT, 10/12/1999

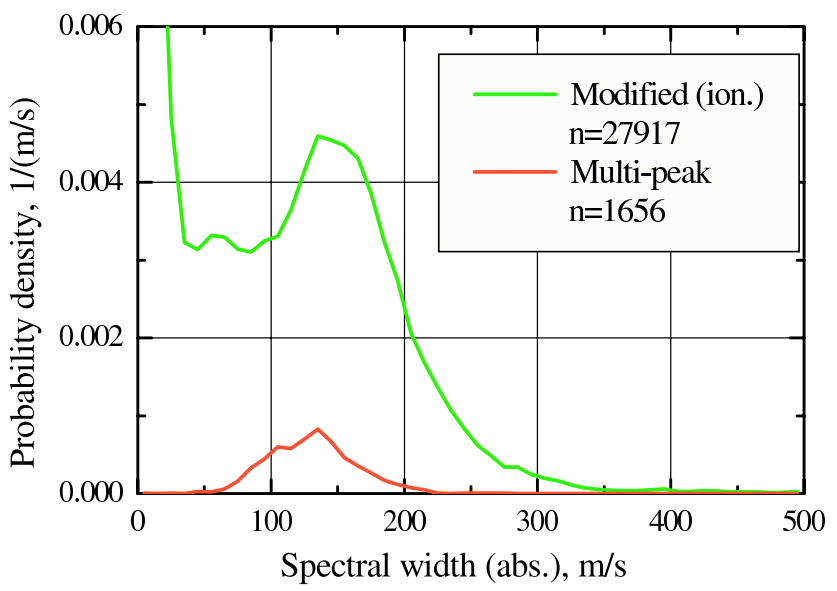

Fig. 12. The same data as in Fig. 7 (green line) but without sea scatter data.

4. To disable the "bad shape" routines dealing with ACF power depletions from an unknown origin.

After applying these modifications, we have obtained timerange maps for spectral width which do not differ much from those shown in Fig. 6 (see, e.g. Fig. 11). Therefore, the major changes compared with the original FITACF can be attributed to retaining $\sigma_{R}$ (red curves in Figs. 7, 8). However, the other modifications further improved separation between low and high width distributions, as well as spectral width error estimates (green curves in the same figures).

\section{Physical mechanisms for large spectral widths}

The results obtained from the modified FITACF, as described in the previous section, have important implications for possible explanations for large spectral width values. Physical mechanisms outlined in the Introduction that could not give spectral width values larger than $\simeq 200 \mathrm{~m} / \mathrm{s}$ can now be reconsidered. Spectral width values obtained from the original FITACF package are considerably overestimated, while those obtained from the modified FITACF routines are significantly smaller and agree quite well with theoretical estimates for Bohm diffusion. The diffusion coefficient from Eq. (1) lies within $150-200 \mathrm{~m}^{2} / \mathrm{s}$, leading to spectral widths of $W \sim 150-200 \mathrm{~m} / \mathrm{s}$. The more strict treatment of CRI discussed in Sect. 3 also requires revision of previous results regarding multi-component spectra obtained via a Fourier transform of the experimental ACFs by Baker et al. (1995) and Moen et al. (2000).

According to collective scatter theory (Rytov et al., 1988), the major contribution to the scattered HF signal at the reception point is provided by random spatial arrays of irregularities with a spatial period of $l_{\|}=\lambda / 2$, according to the Bragg scatter condition. The $\|$ symbol indicates the direction 
parallel to the HF wave vector. The longer these structures exist as a whole and maintain their spatial structure, the larger the correlation time of the scattered field. There are two limiting cases to consider. If the irregularity Lagrangian lifetime (lifetime in the irregularity reference frame, Villain et al., 1996), $T_{L}$, is infinite, the only process causing decorrelation is random velocity fluctuations ("wandering" irregularities). Due to these fluctuations, the resonant arrays will randomly appear and disappear on a characteristic time scale, $T_{V} \sim l_{\|} / \delta V$, where $\delta V$ is the (root mean square) fluctuation of the velocity of the plasma turbulent motion. Physically, $T_{V}$ is the time required for an average irregularity to randomly "wander" across its own scale size $l_{\|}$. In this case the irregularity region ceases to satisfy the Bragg condition, and its scattered field becomes decorrelated.

At the other extreme, if the irregularities are not randomly moving, then lifetime processes are dominant. $T_{L}$ represents a sum of generation time, dwell time (if any) and decay time of an average irregularity with $l_{\|}=\lambda / 2$. Usually, the major contribution to $T_{L}$ is given by the decay time due to plasma diffusion processes, $T_{D}=1 / k^{2} D$, where $D$ is the diffusion coefficient and $k=2 \pi / l_{\|}$. In reality, the correlation time scale, $\tau_{c}$ is determined by the fastest process, so $\tau_{c} \sim \min \left(T_{V}, T_{L}\right)$. To determine the dominant process, it is convenient to introduce a Lagrangian length, $l_{L}=\delta V \cdot T_{L}$, which is the distance travelled by an irregularity during its lifetime due to random velocity fluctuations. If $l_{L} \ll l_{\|}$, then $\tau_{c} \simeq T_{D}$, otherwise $\tau_{c} \simeq T_{V}$.

The most advanced analytical treatment of radar ACFs was given by Hanuise et al. (1993) and Villain et al. (1996). They derived a theoretical expression accounting for both velocity fluctuation and turbulent diffusion processes

$R(\tau)=R(0) e^{-k^{2} D_{t} T_{L}\left(\tau / T_{L}+e^{-\tau / T_{L}}-1\right)}$,

where $D_{t}=\delta V^{2} T_{L}$ is the turbulent diffusion coefficient. Figure 10 shows the dependence of this model on the Lagrangian lifetime.

At short lags, $\tau \ll T_{L}$, the model curve has a Gaussian shape while at longer lags, $\tau \gg T_{L}$, it becomes exponential. The transition between these two shapes occurs at $\tau=2 T_{L}$. The actual shape of the curve is determined by the fastest of the two processes. If $l_{\|} \ll l_{L}$, then after applying a second order Taylor expansion, one obtains a Gaussian ACF,

$R(\tau) \simeq R(0) e^{-\left(k^{2} D_{t} / 2 T_{L}\right) \tau^{2}}$,

with $\tau_{c}=T_{V}=\sqrt{2 T_{L} / k^{2} D_{t}}=\sqrt{2} / k \delta V$. If $l_{\|} \gg l_{L}$, then the ACF becomes exponential,

$R(\tau) \simeq R(0) e^{-k^{2} D_{t} \tau}$,

with $\tau_{c}=T_{D}=1 / k^{2} D_{t}$.

To determine if there was any relationship between the ACF shape and the spectral width boundary we used ionospheric scatter echoes from TIGER for 12:00-14:00 UT on 10 December 1999, as shown in Fig. 11.
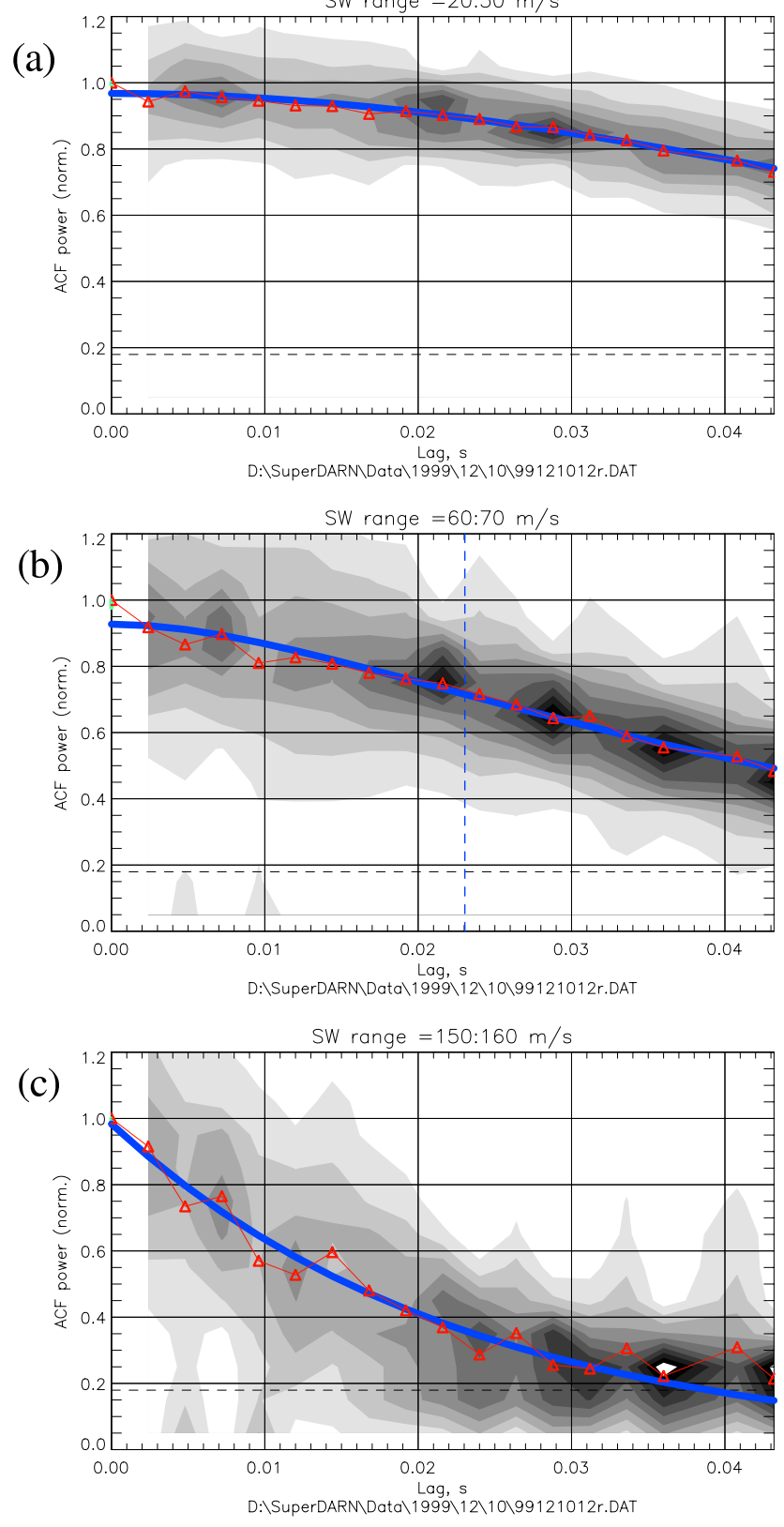

Fig. 13. Median normalised ACF power (red triangles) and the fit to Eq. (2) (blue line). Grey-scale contours show the spread of normalised distribution functions for each lag. Panels (a), (b) and (c) correspond to low $(\mathrm{W}=20-30 \mathrm{~m} / \mathrm{s})$, medium $(\mathrm{W}=60-70 \mathrm{~m} / \mathrm{s})$ and high $(\mathrm{W}=150-160 \mathrm{~m} / \mathrm{s})$ spectral width, respectively.. The vertical dashed line in Fig. 13(b) identifies the transition from a Gaussian to exponential shape at $\tau=2 T_{L}$ (see text for details).

The horizontal dashed line corresponds to the fluctuation level, $\sigma_{R}$.

The empirical criteria in FITACF used to reject sea scatter echoes were

$|W|-W_{\text {err }}<35 \mathrm{~m} / \mathrm{s}$

and

$|V|-V_{\text {err }}<30 \mathrm{~m} / \mathrm{s}$, 


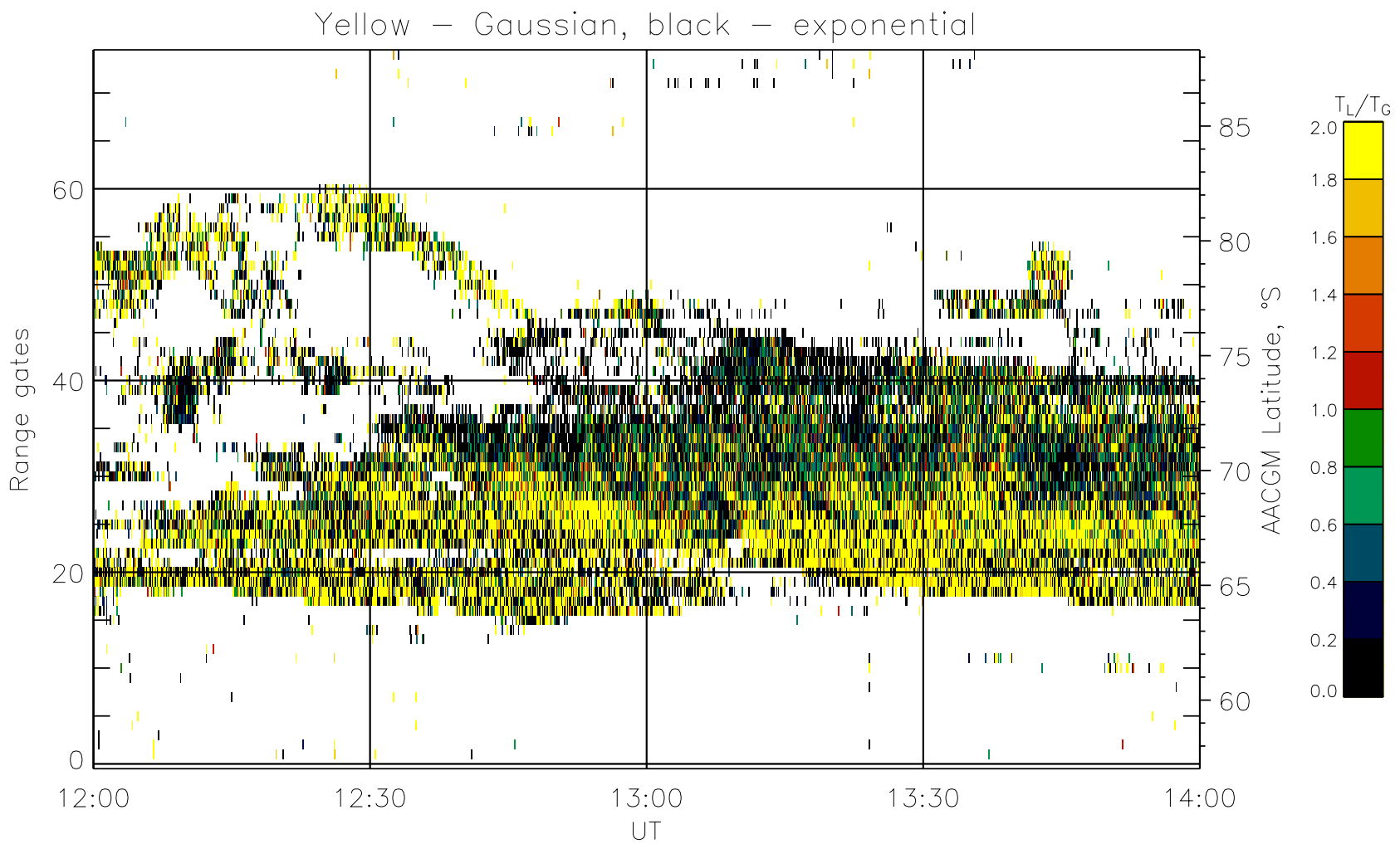

Fig. 14. Ratio between the Lagrangian correlation times, $T_{L}$, and the Gaussian time constant, $T_{G}=T_{V}=1 / k \delta V$, the correlation time related to the random drift of irregularities. Yellow colour corresponds mostly to Gaussian shaped ACF power while black shading depicts mostly the exponential shape.

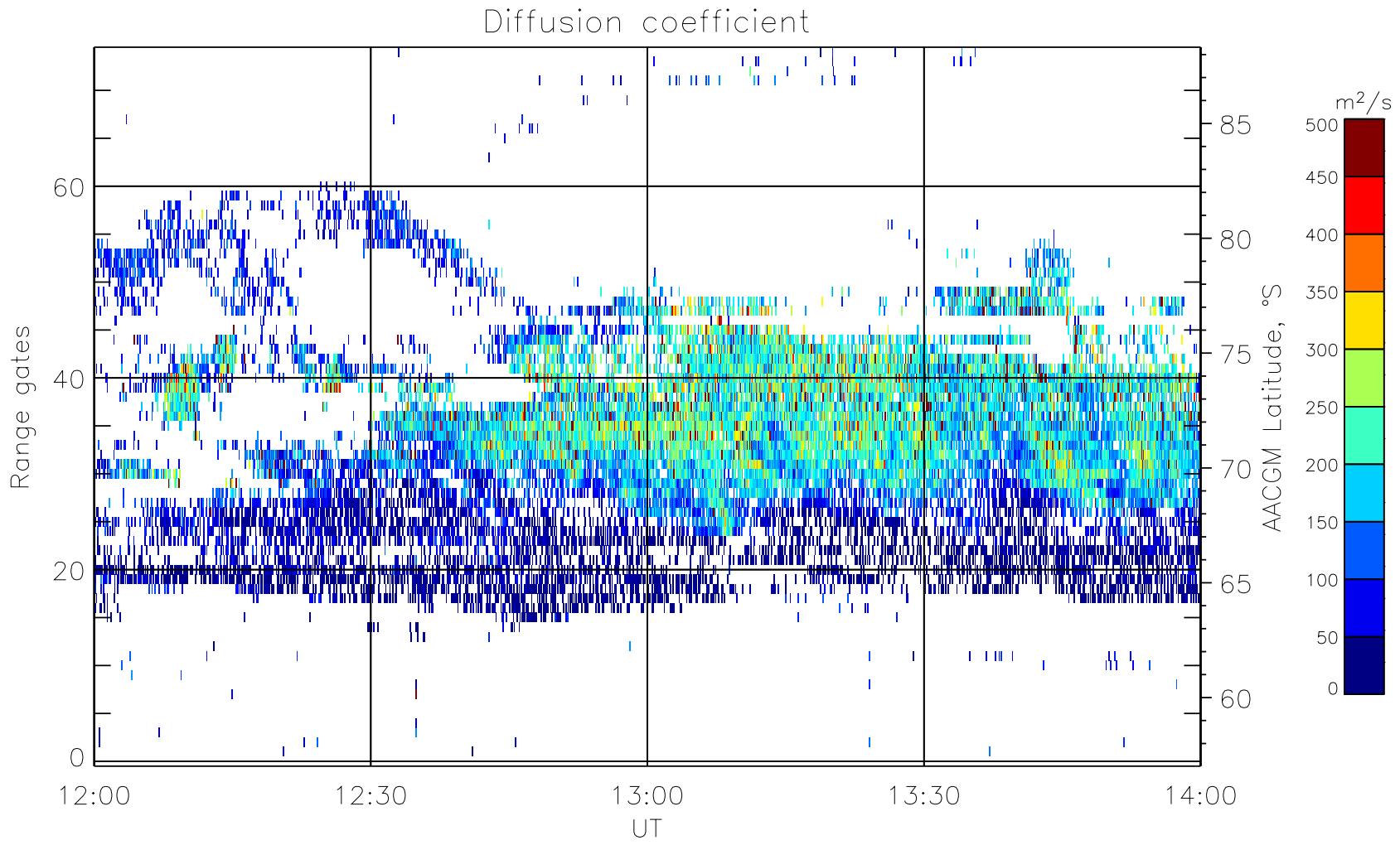

Fig. 15. Effective diffusion coefficient estimated by fitting Eq. (2) to the experimental ACF power (ionospheric scatter). 
where $W_{\text {err }}$ and $V_{\text {err }}$ are the fitting errors for the spectral width and velocity, respectively. The resulting histogram for $W$ is shown in Fig. 12.

Following Villain et al. (1996), we applied Eq. 2 to the same data set. First, we calculated the median normalised ACF power across the three spectral width ranges of 20 $30 \mathrm{~m} / \mathrm{s}, 60-70 \mathrm{~m} / \mathrm{s}$ and $150-160 \mathrm{~m} / \mathrm{s}$. These were chosen based on the histogram in Fig. 12 and correspond to low, intermediate and high spectral widths. The results in Fig. 13 clearly show that small spectral widths are associated with a Gaussian ACF power shape, i.e. random drift velocity variations $\delta V$ dominate all other mechanisms, while the large- $W$ ACF power decays exponentially with $\tau$, indicating that diffusion processes are the major contributor to $R(\tau)$. A similar relationship between $W$ magnitude and ACF shape was reported by Hanuise et al. (1993), but their observations were not related to a spectral width boundary.

Figure 14 shows the ratio $T_{L} / T_{V}$, which may be interpreted as an effective shape factor for Eq. (2). Yellow regions $\left(T_{L} \gg T_{V}\right)$ represent Gaussian type ACFs while black regions $\left(T_{L} \ll T_{V}\right)$ depict exponential ones, with the rest of the palette corresponding to intermediate cases. The larger values for $W$ and predominantly exponential ACFs concentrate poleward of the boundary, while the smaller values for $W$ and the Gaussian-shaped ACFs are generally observed equatorward of the boundary.

The spatio-temporal map of the diffusion coefficient, $D$, is shown in Fig. 15. The values for $D$ are shown for ACFs whose power at the transition lag, $\tau=2 T_{L}$, was above the fluctuation level, $R\left(2 T_{L}\right)>\sigma_{R}$ (Fig. 10). As expected, the spatio-temporal distribution of $D$ in Fig. 15 looks similar to that of $W$ in Fig. 11, due to the simple fact that $W \sim 1 / \tau_{c} \sim k^{2} D$. The corresponding histogram for $D$ is shown in Fig. 16.

Remarkably, the diffusion coefficient is confined to values, $D \leq 200-250 \mathrm{~m}^{2} / \mathrm{s}$, i.e. close to the Bohm diffusion estimate.

Random drift velocity spread (Fig. 17) clearly shows that the velocity fluctuations poleward of the spectral width boundary are substantially larger than those equatorward of the boundary. Here we only use values corresponding to the well-defined Gaussian part of the fitting curve, for example, $R\left(2 T_{L}\right)>1-\sigma_{R}$ (Fig. 10). The corresponding histogram is shown in Fig. 18, and it has the main maximum around $10 \mathrm{~m} / \mathrm{s}$. However, the curve extends well beyond $50 \mathrm{~m} / \mathrm{s}$.

Finally, we investigated possible effects of multicomponent ACF (multi-peak spectra) on the estimates for spectral width (Baker et al., 1995; Moen et al., 2000). First, we designed criteria to distinguish between singlecomponent and multi-component ACFs using the fluctuation level, $\sigma_{R}$. The criteria were based on the fact that two or more spectral components produce interference minima and maxima in ACF power, and only the components comparable in magnitude might cause significant fading of the power and affect $W$. As illustrated in Fig. 19, we identify multi component ACFs as those with power that after descending to $R(\tau) \leq \sigma_{R}$ at smaller lags, grows back to at least $2 \sigma_{R}$ at larger lags.

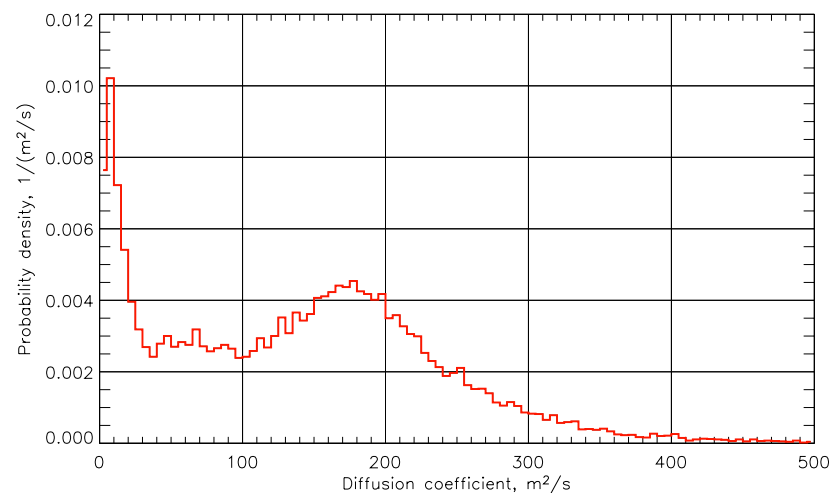

Fig. 16. Histogram for diffusion coefficient data presented in this figure.

After applying this criterion to the TIGER data for 10 December 1999, we found that only a small fraction of ACFs were identified as multi-component (red line in Fig. 12), contributing to $\simeq 6 \%$ of the overall ACFs and not exceeding $20 \%$ at individual histogram bins. The above criterion is rather liberal because the fluctuation level $\sigma_{R}$ may, in fact, be greater than $1 / \sqrt{N_{\text {avg }}}$, due to the presence of CRI, as discussed in Sect. 3.3.

\section{Conclusions}

There have been many unsuccessful attempts to physically interpret large spectral width values $W \geq 250-300 \mathrm{~m} / \mathrm{s}$ measured by SuperDARN radars at high latitudes. We have approached this problem by examining the data processing procedures. A critical revision of the FITACF package that is used for calculating spectral width and other signal parameters at all radar sites revealed inadequate pre-processing of experimental ACFs before using them to estimate $W$. A revised FITACF package was tested against a representative 2-h data set, which contains a pronounced night-time spectral width boundary. The most important result of the software revision was a decrease in the measured spectral width values. While the spectral width boundary location remained unchanged, corrected $W$ were smaller by $20-40 \%$ and confined mostly to $200 \mathrm{~m} / \mathrm{s}$. The smaller $W$ values provided estimates of the effective diffusion coefficient $D \simeq 200-250 \mathrm{~m}^{2} / \mathrm{s}$ poleward of the spectral width boundary. We consider these values to be in satisfactory agreement with the Bohm diffusion mechanism, which was previously dismissed based on overestimated values for $W$ (André et al., 2000). Further analysis showed that the shape of the ACF power poleward of the spectral width boundary (large $W$ ) is mostly exponential, while equatorward of the boundary it is predominantly Gaussian. Contributions from the multicomponent ACFs appeared to be insignificant, at least for our night-time data set. This implies that in the large- $W$ region the scatter ACF is affected mainly by diffusion processes, 


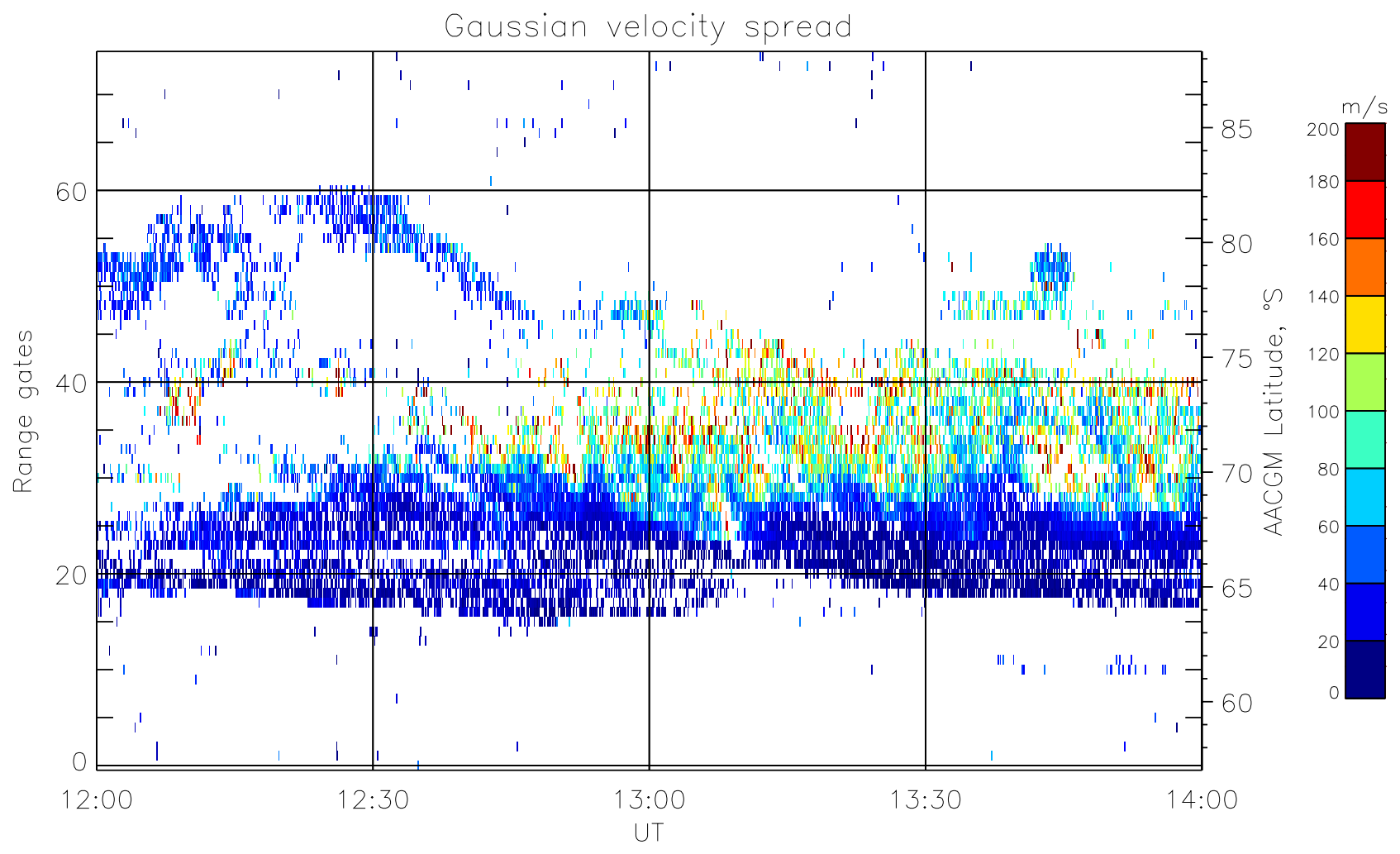

Fig. 17. Range-time map of the random velocity variation determined from Gaussian-shaped ACFs.

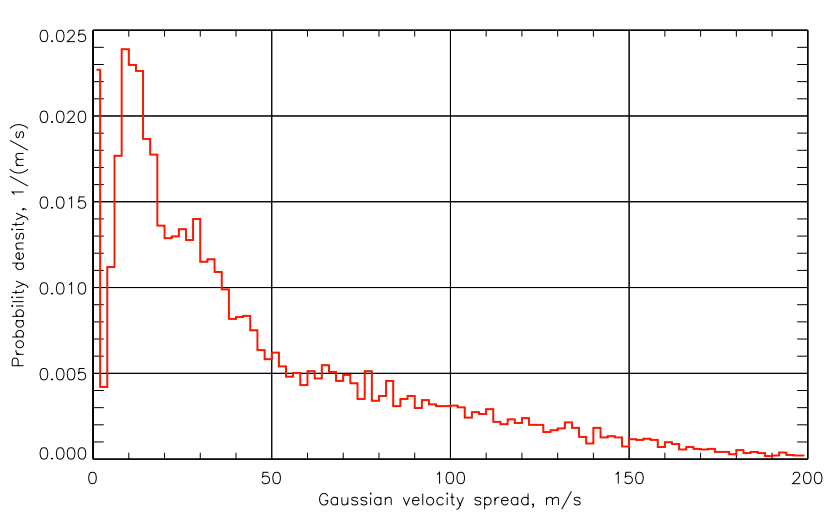

Fig. 18. Histogram of random velocity variations shown in Fig. 17.

while in small- $W$ areas $|R(\tau)|$ is shaped by fluctuations of the plasma drift velocity.

While we have presented a limited data set to illustrate the effect of the modifications to FITACF, we have also analysed other data intervals with very similar results. Further studies will focus on comparisons between night- and daytime mechanisms for large spectral widths and the relationship with the energy spectrum of particle precipitation. The physical mechanisms associated with Bohm diffusion are still unclear, and one of its possible causes might lie in acceleration of plasma transport by unstable plasma waves (Chen, 1984), which are regularly observed in the ionosphere. Studies of

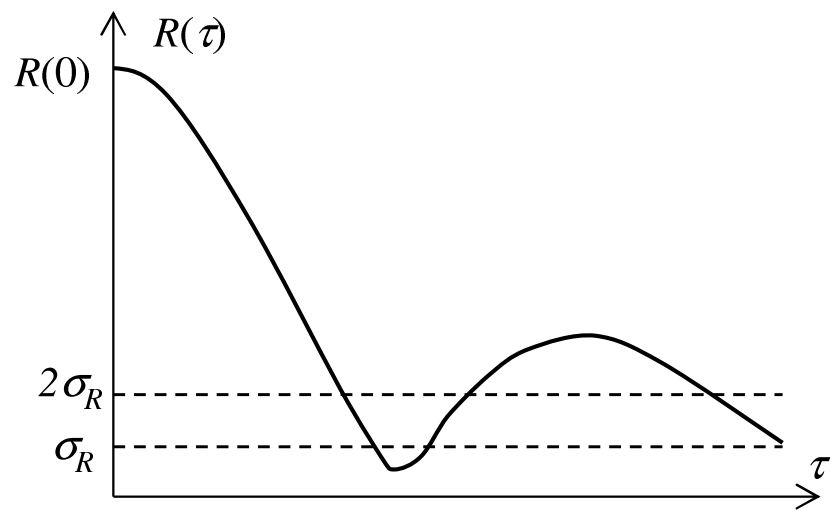

Fig. 19. Illustration of the criterion used for identifying multicomponent ACFs.

$W$ from SuperDARN may be valuable in providing the necessary insights into the details of Bohm diffusion.

\section{Appendix A FITACF routines}

The FITACF software used by all SuperDARN radars consists of a number of subroutines, and the flow chart is presented in Fig. A1. For each beam and time, up to 75 ACFs are obtained, one for each range gate, starting normally from $180 \mathrm{~km}$ and reaching out to $3550 \mathrm{~km}$. These "raw" ACFs are passed to the BADLAGS routine which examines each ACF 
for bad lags that are affected by transmitter pulse overlap or cross-range interference. Next, the background noise level is estimated (NOISE_STAT), and any coherent interference signal is detected and removed by NOISE_ACF, FIT_NOISE and REMOVE_NOISE. The pre-processed ACFs are passed to the MORE_BADLAG routine which identifies bad lags based on the background noise level, statistical fluctuation level, the presence of spurious spikes and assumptions involving decreasing ACF power with lag. After this "clean-up", the ACF power and spectral width, $W$, is estimated by fitting Gaussian and exponential models to the ACF envelope. The Doppler velocity, $V_{D}$, is determined from a linear fit to the ACF phase. Routines ELEVATION and GROUND_SCATTER determine elevation angle of arrival for the HF signal and ground/sea scatter ACFs (for sea/ground scatter selection criteria see Sect. 4), respectively.

\section{A1 Statistical fluctuation level}

The statistical fluctuation level, $\sigma_{R}$, is calculated in the FIT_ACF routine and extracted from $|R(\tau)|$ before fitting. The statistical fluctuation value is derived from the zero lag power divided by the square root of $N_{\text {avg }}$, i.e. $\sigma_{R}=R(0) / \sqrt{N_{\text {avg }}}$. Theoretically, the normalised statistical error level is given by (e.g. Bendat and Piersol, 1986)

$\epsilon_{R}(\tau)=\sqrt{\left[1+R^{-2}(\tau)\right] / 2 N_{\text {avg }}}$,

so that $\sigma_{R}=\epsilon_{R}|R(\tau)|=\sqrt{\left[R^{2}(\tau)+1\right] / 2 N_{\text {avg }}}$ varies between $1 / \sqrt{2 N_{\text {avg }}}$ and $1 / \sqrt{N_{\text {avg }}}$ for $|R(\tau)|=0$ and 1 , respectively. Thus, the FIT_ACF routine uses the maximum estimate $\sigma_{R}(R=1)$.

\section{A2 Noise level}

These FITACF routines are designed to identify which lags of an ACF adversely suffer from different types of noise or interference. These are zero lag noise and non-zero lag noise.

Zero lag noise, $R_{n}(0)$, is the larger of the "clearsky search" noise (passive reception with the transmitter switched off) compared with the average zero lag power obtained from the 10 weakest ACFs along a beam direction. This parameter is calculated in NOISE_STAT and used to reject ACFs with small zero lag signal to noise ratio and to calculate the non-zero lag noise.

Non-zero lag noise (NOISE_STAT) is obtained by identifying all ACFs for a given beam that have zero lag power, $R(0)$, less than $1.6 R_{n}(0)+\sigma_{R}$ and then calculating the average non-zero lag noise power, $\left\langle R_{n}(\tau \neq 0)\right\rangle$, from all these identified ACFs. This is a single number obtained via summing power from all non-zero lags together and dividing by their total number. In FITACF $\left\langle R_{n}(\tau \neq 0)\right\rangle$ is subtracted from $|R(\tau)|$ in all range gates before fitting.

\section{A3 "Bad lag" routines}

The two routines that identify and deal with "bad lags" are BADLAGS and MORE_BADLAGS. The BADLAGS

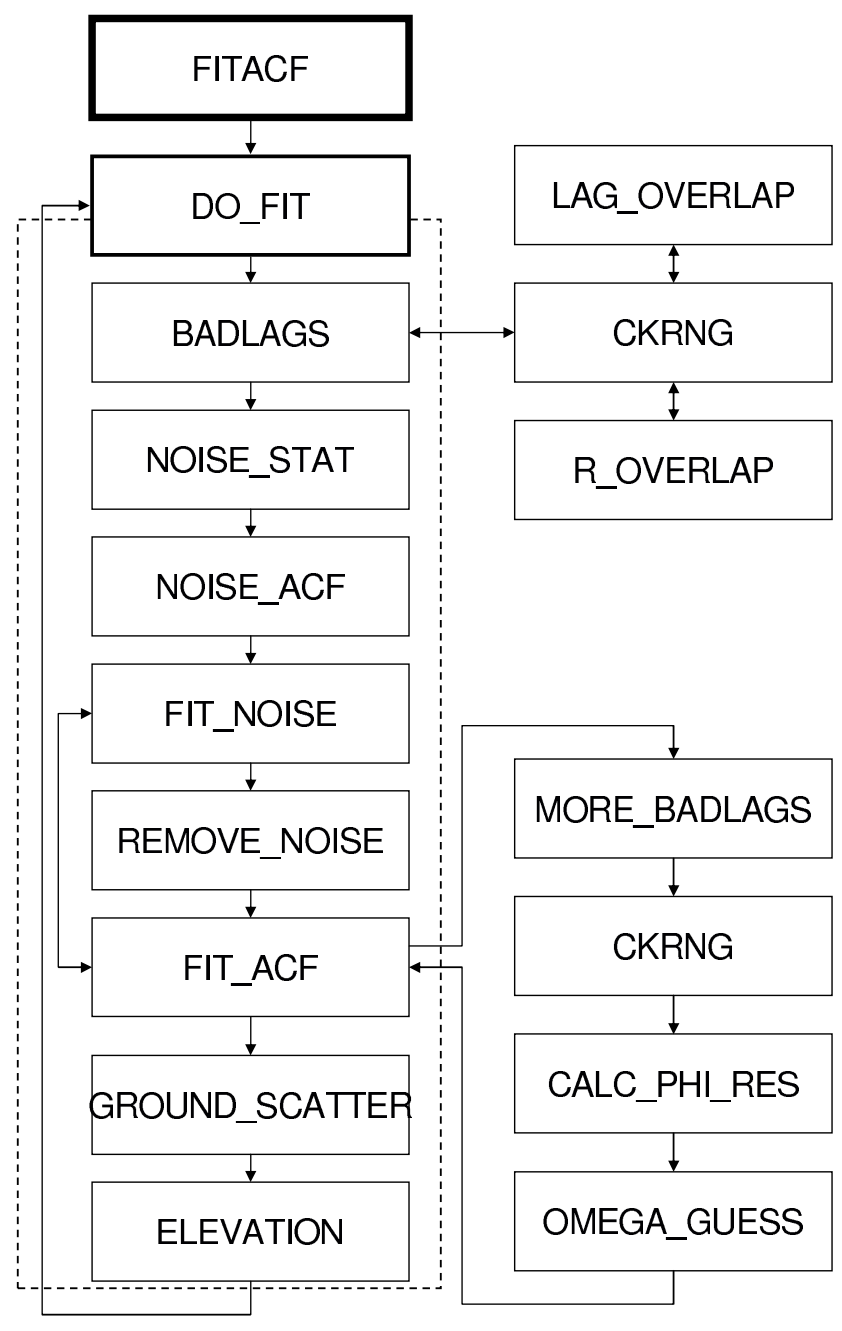

Fig. A1. Flow chart of FITACF procedures.

routine marks ACF lags contaminated by transmit pulse overlap and cross-range interference. For cross-range interference, the zero lag power of the current $\mathrm{ACF}, R_{\text {cur }}(0)$, is compared with the zero lag power of the ACF from the potentially interfering range, $R_{\text {int }}(0)$. If $R_{\text {int }}(0)$ is sufficiently large, the corresponding interfering lags are marked as "bad". The empirical threshold used for this purpose is $\mu=R_{\text {int }}(0) / R_{\text {cur }}(0)=0.3 N_{\text {avg }}$.

The MORE_BADLAGS routine is designed to adjust the ACF according to a single-component shape assumed by the fitting routines. Importantly, before this the fluctuation level, $\sigma_{R}$, and non-zero lag noise, $\left\langle R_{n}(\tau \neq 0)\right\rangle$, are extracted from $|R(\tau)|$, which may lead to negative values for ACF power. ACF lags are marked as "bad" according to the following rules:

- ACF power is less than zero, $|R(\tau)| \leq 0$.

- If two consecutive points in the ACF power are less than zero, then the longer lags ("tail") are dismissed: 


$$
\begin{aligned}
& R\left(\tau_{i}\right)<\left\langle R_{n}(\tau \neq 0)\right\rangle \text { and } R\left(\tau_{i+1}\right)<\left\langle R_{n}(\tau \neq 0)\right\rangle \rightarrow \\
& R\left(\tau_{j}\right) \equiv \text { "bad" for } j>i+1 .
\end{aligned}
$$

- If the ACF power increases with increasing lag outside the uncertainty limit, determined by the fluctuation level, and is twice the non-zero lag noise, then this lag is declared as "bad":

$$
\begin{aligned}
& R\left(\tau_{i}\right)>R\left(\tau_{i-1}\right)+R(0) / \sqrt{2 N_{\text {avg }}}+2\left\langle R_{n}(\tau \neq 0)\right\rangle \rightarrow \\
& R\left(\tau_{i}\right) \equiv \text { "bad". }
\end{aligned}
$$

This effectively removes positive "spikes" in the ACF power and forces for the decaying shape to comply with the single component fitting models. However, if the following lag is "well-behaved", then the previous lag is considered as a negative "spike", and the current lag is relabelled as "good":

$$
\begin{aligned}
& R\left(\tau_{i+1}\right)<R\left(\tau_{i}\right)+R(0) / \sqrt{2 N_{\text {avg }}}+2\left\langle R_{n}(\tau \neq 0)\right\rangle \rightarrow \\
& R\left(\tau_{i-1}\right) \equiv \text { "bad", } \\
& R\left(\tau_{i}\right) \equiv \text { "good". }
\end{aligned}
$$

Acknowledgements. This work was supported by the Australian Research Council, the University of Newcastle, and the Cooperative Research Centre for Satellite Systems through the Commonwealth of Australia CRC Program. We also thank P. Dyson, J. Devlin and M. Parkinson for operating and providing data from the TIGER radar. We would like to thank K. Baker for fruitful discussions.

Topical Editor M. Pinnock thanks R. A. Greenwald and M. Freeman for their help in evaluating this paper.

\section{References}

André, R., Pinnock, M., and Roger, A. S.: On the SuperDARN autocorrelation function observed in the ionospheric cusp, Geophys. Res. Lett., 26, 3353-3356, 1999.

André, R., Pinnock, M., Villain, J. P., and Hanuise, C.: On the factor conditioning the Doppler spectral width determined from SuperDARN HF radars, Int. J. Geomagn. Aeron., 2, 77-86, 2000.

Baker, K. B., Dudeney, J. R., Greenwald, R. A., Pinnock, M., Newell, P. T., Rodger, A. S., Mattin, N., and Meng, C.-I.: HF radar signatures of the cusp and low-latitude boundary layer, J. Geophys. Res., 100, 7671-7695, 1995.

Barthes, L., André, R., Cerisier, J.-C., and Villain, J.-P.: Separation of multiple echoes using a high-resolution spectral analysis for SuperDARN HF radars, Radio Sci., 33, 1005-1017, 1998.

Bendat, J. S. and Piersol, A. G.: Random data, Analysis and measurement procedures, John Wiley \& Sons, Inc., New York, Chichester, Brisbane, Toronto and Singapore, 1986.

Chen, F. F.: Introduction to plasma physics and controlled fusion, second edition, vol. 1: Plasma Physics, Plenum Press, New York and London, 1984.
Chisham, G., Freeman, M. P., and Sotirelis, T.: Statistical comparison of SuperDARN spectral width boundaries and DMSP particle precipitation boundaries in the nightside ionosphere, Geophys. Res. Lett., 31, L02804, doi:10.1029/2003GL019074, 2004

Chisham, G., Freeman, M. P., Sotirelis, T., Greenwald, R. A., Lester, M., and Villain, J.-P.: A statistical comparison of SuperDARN spectral width boundaries and DMSP particle precipitation boundaries in the morning sector ionosphere, Ann. Geophys., 23, 733-743, 2005,

SRef-ID: 1432-0576/ag/2005-23-733.

Dudeney, J. R., Rodger, A. S., Freeman, M. P., Pickett, J., Scudder, J., Sofko, G., and Lester, M.: The nightside ionospheric response to IMF $B_{y}$ changes, Geophys. Res. Lett., 25, 2601-2604, 1998.

Greenwald, R. A., Baker, K. B., Hutchins, R. A., and Hanuise, C.: An HF phased-array radar for studying small-scale structure in the high-latitude ionosphere, Radio Sci., 20, 63-79, 1985.

Greenwald, R. A., Baker, K. B., Dudeney, J. R., Pinnock, M., Jones, T. B., Thomas, E. C., Villain, J. P., Cerisier, J. C., Senior, C., Hanuise, C., Hunsucker, R. D., Sofko, G., Koehler, J., Nielsen, E., Pellinen, R., Walker, A. D. M., Sato, N., and Yamagishi, H.: DARN/SuperDARN: A global view of the dynamics of highlatitude convection, Space. Sci. Rev., 71, 761-795, 1995.

Hanuise, M., Villain, J. P., Gresillon, D., Cabrit, B., Greenwald, R. A., and Baker, K. B.: Interpretation of HF radar ionospheric Doppler spectra by collective wave scattering theory, Ann. Geophys., 11, 29-39, 1993.

Hysell, D. L., Kelley, M. C., Yampolski, Y. M., Beley, V. S., Koloskov, A. V., Ponomarenko, P. V., and Tyrnov, O. F.: HF radar observations of decaying artificial field-aligned irregularities, J. Geophys. Res., 101, 26 981-26 993, 1996.

Moen, J., Carlson, H. C., Milan, S. E., Shumilov, N., Lybekk, B., Sandholt, P. E., and Lester, M.: On the collocation between dayside auroral activity and coherent HF radar backscatter, Ann. Geophys., 18, 1531-1549, 2000,

SRef-ID: 1432-0576/ag/2000-18-1531.

Parkinson, M. L., Chisham, G., Pinnock, M., Dyson, P. L., and Devlin, J. C.: Magnetic local time, substorm, and particle precipitation-related variations in the behaviour of SuperDARN Doppler spectral width, Ann. Geophys., 22, 4103-4122, 2004,

SRef-ID: 1432-0576/ag/2004-22-4103.

Ponomarenko, P. V. and Waters, C. L.: The role of Pc1-2 waves in spectral broadening of SuperDARN echoes from high latitudes, Geophys. Res. Lett., 30, L1122, doi:10.1029/2002GL016333, 2003.

Ponomarenko, P. V., Menk, F. W., Waters, C. L., and Sciffer, M. D.: Pc34 ULF waves observed by the SuperDARN TIGER radar, Ann. Geophys., 23, 1271-1280, 2005,

SRef-ID: 1432-0576/ag/2005-23-1271.

Rytov, S. M., Kravtsov, Y. A., and Tatarskii, V. I.: Principles of Statistical Radiophysics, Springer-Verlag, New York, 1988.

Valliéres, X., Villain, J.-P., Hanuise, C., and André, R.: Ionospheric propagation effects on spectral widths measured by SuperDARN HF radars, Ann. Geophys., 22, 2023-2031, 2004,

SRef-ID: 1432-0576/ag/2004-22-2023.

Villain, J.-P., André, R., Hanuise, C., and Gresillon, D.: Observation of the high-latitude ionosphere by HF radars: Interpretation in terms of collectivewave scattering and characterization of the turbulence, J. Atmos. Sol. Terr. Phys., 58, 943-958, 1996.

Yukimatu, A. S. and Tsutsumi, M.: A new SuperDARN meteor wind measurement: Raw time series analysis method and its application to mesopause region dynamics, Geophys. Res. Lett., 29, 1981, doi:10.1029/2002GL015 210, 2002. 